\title{
Thermal-wave interferometry of gas-liquid applied to a thermal-wave resonator cavity technique
}

\begin{abstract}
We propose the potential use of thermal-wave interferometry in measuring thermal diffusivities of two media with a thermal-wave resonator cavity technique. In the derived expression for a two-layer configuration, during cavity length probing of the upper medium, the physical and thermal properties of the lower medium were reasonably assumed to be independent of cavity length; hence each of the two slopes of signal versus cavity length actually determines the thermal diffusivity of the corresponding medium. In order to check the validity of the proposed model, we measure the thermal diffusivity of air and glycerol. A good linear relation of the amplitude and the phase with respect to cavity length in the thermally thick region of both media was observed, and the thermal diffusivities of air and glycerol obtained were close to the literature values. We suggest the potential application of thermal-wave resonator cavity technique to measure the thermal properties of a single-layer fluid in a general thermal condition where the advantage is that the signal-to-noise ratio is normally high compared to the thermally thick case. This can be achieved possibly by coating the pyroelectric transducer with a thermally thick solid material prior to any measurements.
\end{abstract}

